\title{
The superburst recurrence time in luminous persistent LMXBs
}

\author{
L. Keek ${ }^{1,2,4}$, J. J. M. in 't Zand ${ }^{1,2}$, and A. Cumming ${ }^{3}$ \\ 1 SRON Netherlands Institute for Space Research, Sorbonnelaan 2, 3584 CA Utrecht, The Netherlands \\ e-mail: 1.keek@sron.nl \\ 2 Astronomical Institute, Utrecht University, Princetonplein 5, 3584 CC Utrecht, The Netherlands \\ 3 Physics Department, McGill University, 3600 rue University, Montreal, QC, H3A 2T8, Canada \\ Received 20 January 2006 / Accepted 11 May 2006
}

ABSTRACT

\begin{abstract}
Theory and observations favor stable helium burning as the most important means to produce fuel for superbursts on neutron star surfaces. However, all known superbursters exhibit unstable burning as well. This ambiguity prompted us to search for superbursts in data from the BeppoSAX Wide Field Cameras of ten luminous LMXBs, most of which do not exhibit normal type-I X-ray bursts. We found no superbursts and determine a lower limit on the recurrence time which varies between 30 and 76 days (90\% confidence). All recurrence time limits except one are longer than the observed recurrence time for GX 17+2. This difference can be understood if the mass accretion rate in GX $17+2$ is several tens of percent higher than in the other sources; alternatively, the accreted material in GX 17+2 might be hydrogen deficient, leading to larger carbon yields than in the other sources. We compare our results to the latest models of superbursts. As our search method is indiscriminate of the burst ignition scenario, the recurrence time limits may also be applied to other bursts of similar duration and brightness.
\end{abstract}

Key words. stars: neutron $-\mathrm{X}$-rays: binaries $-\mathrm{X}$-rays: bursts

\section{Introduction}

Neutron stars in low-mass X-ray binaries (LMXBs) accrete hydrogen- and helium-rich matter from a sub-solar mass companion star. The gravitational energy that is released when the matter travels to the surface of the neutron star accounts for most of the observable X-ray radiation. The accreted matter forms a layer on the neutron star. When this layer is sufficiently compressed and heated, thermonuclear burning is ignited. The burning can be stable or unstable, depending on e.g. the mass accretion rate. Unstable nuclear burning results in a type I X-ray burst (Grindlay et al. 1976; Belian et al. 1976; Woosley \& Taam 1976; Maraschi \& Cavaliere 1977). A burst is observed as a fast (1-10 s) rise in the flux and a slow (10-1000 s), exponentiallike decay. Many such bursts have been observed from about 80 sources (see e.g. in 't Zand et al. 2004b). For reviews see Lewin et al. (1993) and Strohmayer \& Bildsten (2003).

Longer X-ray bursts have been observed that have a similar fast rise, but a much longer decay of several hours. These so-called superbursts release about a thousand times more energy than the normal type I X-ray bursts. So far, ten superbursters have been identified, with two exhibiting more than one superburst. For an observational overview, see Kuulkers (2004) (and see also in 't Zand et al. 2004a; Remillard et al. 2005; and Kuulkers 2005, for reports of new superbursters).

Superbursts are ascribed to unstable carbon burning (initiated by ${ }^{12} \mathrm{C}+{ }^{12} \mathrm{C} \rightarrow{ }^{20} \mathrm{Ne}+\alpha$ ) in an "ocean" of heavy elements which lies between the freshly accreted layer and the neutron star crust (Cumming \& Bildsten 2001; Strohmayer \& Brown 2002). The carbon and heavy elements are thought to be the products of rp-process hydrogen and helium burning in the accreted layer (see e.g. Schatz et al. 1999, 2003). This model has been fairly successful at reproducing the energetics and recurrence times of superbursts
(Cumming \& Bildsten 2001; Strohmayer \& Brown 2002; Brown 2004; Cooper \& Narayan 2005; Cumming et al. 2005), as well as the observed lightcurves and the quenching of normal type I X-ray bursts for about a month following a superburst (Cumming \& Macbeth 2004; Cumming et al. 2005).

An open issue is how to produce enough carbon to power a superburst. Carbon fractions of more than $10 \%$ are required to reproduce the observed lightcurves of superbursts and to achieve unstable ignition at the accretion rates of approximately $0.1 \dot{M}_{\text {Edd }}$ inferred for most of the superbursters ( $\dot{M}_{\text {Edd }}$ is the Eddington accretion rate). Calculations of rp-process burning show that stable rather than unstable $\mathrm{H} / \mathrm{He}$ burning is required for such large carbon fractions (Schatz et al. 2003). The reason is that carbon production is only possible by helium burning after the hydrogen has been consumed, since hydrogen readily captures on carbon, processing it to heavy elements. Helium is rapidly consumed at the high temperatures reached during unstable burning. However, in stable burning, the helium burns at a reduced rate so that when the hydrogen has been completely consumed by rp-process burning, there is still some helium left that then burns into carbon. In this way, there is an anti-correlation between the amount of carbon that is produced and the length of the rp-process, or equivalently the average mass of the heavy elements (Schatz et al. 2003).

Observations support the presence of stable burning in superburst sources. In 't Zand et al. (2003) showed that superburst sources preferentially have large values of alpha, the ratio of the persistent fluence between type I X-ray bursts to the fluence in the type I X-ray burst. Large values of alpha indicate that not all the nuclear fuel is consumed in bursts, and that stable burning must occur between them. Cornelisse et al. (2003) studied BeppoSAX observations of nine type I X-ray bursters and found that the burst rate drops for luminosities exceeding approximately $10 \%$ of the Eddington limit, implying that stable burning 
Table 1. Superburst recurrence time of ten sources. For each source we give the time in days between the first and the last observation $t_{\mathrm{span}}$, the total observation time $t_{\mathrm{obs}}$ including data gaps, the flaring fraction, the number of observed superbursts (SBs), whether a source exhibits normal bursts and the (lower limit of the) superburst recurrence time $t_{\text {recur }}$.

\begin{tabular}{llllccl}
\hline \hline Source & $t_{\text {span }}[\mathrm{d}]$ & $t_{\text {obs }}[\mathrm{d}]$ & Flaring fraction & \# SBs & Burster & $t_{\text {recur }}[\mathrm{d}]$ \\
\hline Sco X-1 & 1870.03 & 55.44 & $16 \%$ & 0 & no & $>30$ \\
GX 340+0 & 2062.82 & 153.20 & $10 \%$ & 0 & no & $>74$ \\
GX 349+2 & 2062.82 & 129.71 & $12 \%$ & 0 & no & $>66$ \\
GX 9+9 & 2062.57 & 139.27 & $8 \%$ & 0 & no & $>76$ \\
GX 354-0 & 2062.79 & 100.97 & $10 \%$ & 0 & yes & $>52$ \\
GX 5-1 & 2062.79 & 109.95 & $11 \%$ & 0 & no & $>55$ \\
GX 9+1 & 2062.79 & 103.45 & $9 \%$ & 0 & no & $>53$ \\
GX 13+1 & 2062.79 & 104.30 & $9 \%$ & 0 & yes & $>55$ \\
GX 17+2 & 2062.80 & 127.59 & $16 \%$ & 4 & yes & $30 \pm 15^{a}$ \\
Cyg X-2 & 1999.10 & 109.67 & $11 \%$ & 0 & yes & $>60$ \\
\hline
\end{tabular}

${ }^{a}$ From in 't Zand et al. (2004a).

is occurring. This critical luminosity is close to the lowest luminosity of superburst sources, and may explain why superbursts have not been seen at accretion rates less than $\sim 0.1 \dot{M}_{\text {Edd }}$.

In contrast, the role of unstable burning and the heavy elements produced by the rp-process remains unclear. Observationally, it is true that all superburst sources show type I X-ray bursts. Cumming \& Bildsten (2001) pointed out that for a fixed heat flux emerging from the neutron star crust, the heavy nuclei reduce the thermal conductivity of the accumulating ocean, leading to higher temperatures and earlier carbon ignition than previously found in models of pure carbon layers (see e.g. Woosley \& Taam 1976). However, Brown (2004) showed by considering the thermal state of the entire neutron star that in fact the thermal state of the core and crust of the neutron star is most important for setting the ignition column ${ }^{1}$. Because the flux emerging from the crust changes with the heavy element composition, the overall effect of the heavy elements is much smaller than calculated by Cumming \& Bildsten (2001). Nonetheless, the heavy elements do have some effect on the ignition depth, and so it is possible that unstable burning could contribute to the presence of superbursts.

One way to address this issue is to study the several persistently bright LMXBs which either do not or very rarely show type I X-ray bursts. The rapidly accreting source GX 17+2 has already been important for testing carbon ignition models. Cumming \& Bildsten (2001) showed that superbursts should occur at rapid accretion rates near Eddington, but with reduced energies and recurrence times. In 't Zand et al. (2004a) identified four superbursts from GX 17+2 which well-matched the predicted recurrence times, although their durations were longer than expected. In this paper, we report on a systematic search for superbursts in BeppoSAX Wide Field Camera data of ten LMXBs with sufficiently high accretion levels. This includes the so-called "GX" sources: very bright LMXBs with luminosities close to the Eddington limit. We include six sources that never exhibited an X-ray burst as well as four X-ray bursters for comparison purposes, including the known superburster GX $17+2$. We compare the behavior of the four most luminous sources Sco X-1, GX 340+0, GX 5-1 and Cyg X-2 to the equally luminous superburster GX $17+2$. For all these sources we find a constraining lower limit on the superburst recurrence time.

1 This is a very exciting result because it opens up the possibility of using superburst observations as a probe of the neutron star interior (Brown 2004; Cooper \& Narayan 2005; Cumming et al. 2005).

\section{Observations}

We search for superbursts in archival data from the Wide Field Cameras (WFC, Jager et al. 1997) on BeppoSAX (Boella et al. 1997a) of ten X-ray sources. The two WFCs were identical coded mask telescopes pointing in opposite directions. They had $40^{\circ} \times 40^{\circ}$ fields of view and a $5^{\prime}$ angular resolution in the 2-25 keV bandpass. They were active between 1996 and 2002. The WFCs carried out a program of semi-yearly campaigns on the Galactic center region. For a review see in 't Zand et al. (2004b). We consider nine sources near the Galactic center and Cyg X-2 (see Table 1). The selected sources accrete mass at a comparable rate to the known superbursters. Some of the sources exhibit normal bursts while others do not.

For each source there is 55 to 153 days of observation time accumulated over a period of six years (see Table 1). Note that this is not the net exposure time, but includes data gaps due to Earth occultations (typically 36 min long and recurring every $96 \mathrm{~min}$ ) and passages through the South Atlantic Anomaly (13 to 26 min long). During these data gaps there is no observation of the source. However, the duration of a data gap is short with respect to the expected duration of a superburst. These data gaps are, therefore, not very detrimental to the detection of superbursts. Except for these short datagaps, an uninterrupted period of exposure time is typically 1.5 days long.

To determine the persistent flux in the $0.1-200 \mathrm{keV}$ range of GX $9+9$ we use data from the BeppoSAX Narrow-Field Instruments (NFI). Of these instruments we use data from the Low Energy Concentrator Spectrometer (LECS, Parmar et al. 1997, 0.1-10 keV), the Medium Energy Concentrator Spectrometers two and three (MECS, Boella et al. 1997b, 1.3-10 keV) and the Phoswich Detection System (PDS; Frontera et al. 1997; 13-200 keV). For the other sources we obtain a measure of the flux from the literature (see Sect. 5).

\section{Search for superbursts}

For each source we have 55 to 153 days of observation time while a superburst is typically observable for several hours. From the superbursts thus far detected we know that they are rare, so we expect to find at most a few new superbursts. The search for these consists of an automatic peak-search algorithm and a visual inspection of the data.

We use a lightcurve in the full bandpass at three different time resolutions. The highest time resolution is $16 \mathrm{~s}$. This resolution allows to discriminate between fast and slow rising peaks. A higher time resolution is not useful because the uncertainty 
in each lightcurve point becomes too large. The next time resolution is 0.02 days (about $0.5 \mathrm{~h}$ ). We find this resolution good to resolve superbursts at optimum sensitivity since short features such as normal bursts are averaged out while superbursts are not. The lowest time resolution is $0.1 \mathrm{~d}$. We employ it to measure the non-flaring persistent flux. At this resolution superbursts are largely averaged out while typical variations in the persistent flux are well sampled.

The search algorithm proceeds as follows. At $0.02 \mathrm{~d}$ time resolution we check each data point whether it exceeds the persistent flux significantly. Each data point is assigned a measure of the persistent flux through a linear interpolation of the preceding and following $0.1 \mathrm{~d}$-resolution data point. We then check whether the $0.02 \mathrm{~d}$-resolution data point exceeds the persistent flux at $4.4 \sigma$ significance. We choose $4.4 \sigma$ since at this level of significance we detect all superbursts of GX 17+2 while limiting the number of false peaks. There are typically 6000 points in a $0.02 \mathrm{~d}$-resolution lightcurve. We expect the number of false peaks exceeding $4.4 \sigma$ due to Gaussian noise to be 0.06 .

Using the lightcurve at $16 \mathrm{~s}$ time resolution we check these peaks visually for superburst characteristics: a fast rise and a slow, exponential-like decay.

The lightcurve may contain features that have the characteristics of a superburst but are flares (in 't Zand et al. 2004a). Flares are thought to result from quick changes in the mass accretion rate. They are generally not expected to be isolated events, but to come in multiples during flaring episodes. Therefore we exclude obvious flaring periods. We identify flaring episodes visually from the light curve. The fraction of the lightcurve that shows flaring is presented in Table 1. For the identification of a flaring episode we use a similar argument as in 't Zand et al. (2004a): an interval between two consecutive data points at $0.1 \mathrm{~d}$-resolution is identified as a flaring episode when the root mean square of the count rate at $0.02 \mathrm{~d}$ time resolution in that interval exceeds the average root mean square of all intervals by a factor of two. The flaring fractions are on average $11 \%$.

Using this method we reproduce the four superbursts found previously for GX $17+2$ as well as the two peaks that were determined to be flares (in 't Zand et al. 2004a). For the other sources we find no superbursts and we determine a lower limit on the superburst recurrence time $t_{\text {recur }}$.

\section{Recurrence time}

Thus far only two out of ten superbursters exhibited more than one superburst. These are therefore the only sources for which a recurrence time has been directly obtained. From GX 17+2 four superbursts have been observed with an average recurrence time of $30 \pm 15$ days and from 4U 1636-536 three superburst were observed with recurrence times of 1.75 and 2.9 years (Kuulkers et al. 2004).

To determine the lower limit of the recurrence time for the other nine sources here investigated, we perform Monte Carlo simulations of series of superbursts and cross check those with the WFC observation schedule. We generate the series of onset times, for a given $t_{\text {recur }}$, by first randomly picking the time of the initial superburst between 0 and $t_{\text {recur }}$. Subsequent superburst times are sampled from a Gaussian distribution with a standard deviation $\sigma$, centered around a time $t_{\text {recur }}$ after the previous superburst. We use the Gaussian distribution to model the variability in the recurrence time resulting from the variability of the mass accretion rate as measured through the flux. For the nine sources we find from the normalized root mean squared of the flux that $\sigma$ is between 0.06 and $0.3 t_{\text {recur. }}$.

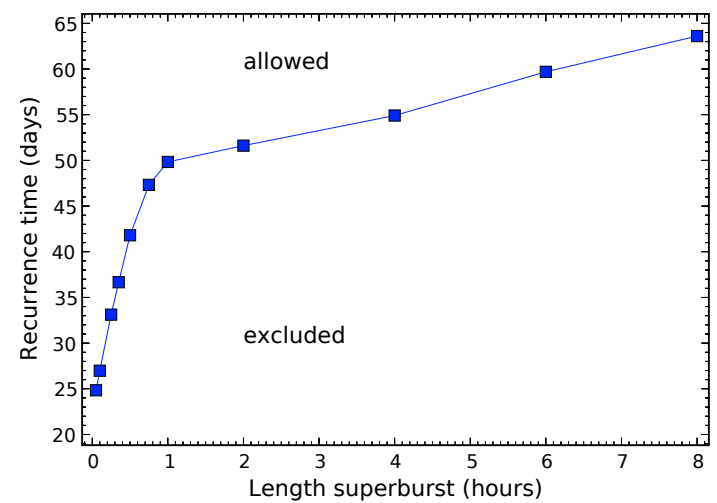

Fig. 1. Lower limit to the recurrence time at $90 \%$ confidence as a function of assumed superburst duration in Monte Carlo simulations. The lower limit drops steeply when the burst duration is of the order of the length of the data gaps or less. Note that our search is insensitive to bursts shorter than $0.5 \mathrm{~h}$ due to our choice of lightcurve time resolution (see Sect. 3).

The superburst exponential decay times are randomly sampled between 1 and $6 \mathrm{~h}$ (see review by Kuulkers 2004). As we will show in more detail below, we can take the decay time independently from the wait time since the previous superburst, because we consider decay times longer than the duration of the data gaps. We assume the peak flux to be equivalent to half the Eddington limit of each source. This implies that any superburst is detectable with the WFC for as long as the e-folding decay time.

A given superburst is considered to be detected if at any time of its e-folding decay duration the instrument was observing the source according to the WFC observation schedule, taking into account all data gaps, including those due to Earth occultations and passages through the South Atlantic Anomaly, as well as flaring episodes.

For each source and $t_{\text {recur }}$ we perform 100000 Monte Carlo simulations. With this number of simulations one expects an uncertainty of $0.3 \%$ in the obtained value. We determine the percentage of simulations in which at least one superburst is detected. We define the lower limit to the recurrence time to be that value of $t_{\text {recur }}$ for which there is a $90 \%$ probability of detecting a superburst. The results are provided in Table 1. We find lower limits for $t_{\text {recur }}$ between 30 and 76 days.

In Fig. 1 we illustrate the dependence of the superburst detection on the duration from the Monte Carlo simulations. For this we extend the duration range to below $1 \mathrm{~h}$. This clearly shows that for superburst durations above $1 \mathrm{~h}$, the presence of short datagaps is not detrimental to the detection of superbursts.

Our aim is to look for superbursts, but in fact our search is not limited to carbon flashes. The search method we use is indiscriminate of the ignition scenario. Other X-ray bursts of sufficient duration and brightness will also be found using our method. Long duration bursts have been observed from several sources (see Cumming et al. 2005; Kuulkers et al. 2002). However, the exponential decay time of these bursts is $\leq 600 \mathrm{~s}$, which is shorter than the lightcurve time resolution of $0.02 \mathrm{~d}$ that we use (see Sect. 3). Therefore we would not have been able to detect these bursts. Note that if a lightcurve at a shorter time resolution is used, such that these long burst are detectable, the limits that can be placed on the recurrence time are far less constraining than for superbursts, since the burst duration is in this case shorter than the data gaps in our observations (see Fig. 1). Nevertheless, the constraints we find on the recurrence time apply not only to superbursts, but to any type of thermonuclear flashes which are long and bright enough to be detected. 
Table 2. Flux and luminosity estimates of the 10 investigated sources. We give the average persistent flux $F$ in the energy range indicated below, the relative variability of the flux as determined from ASM/RXTE measurements, distance $d$, luminosity $L$ in units of the Eddington luminosity $L_{\mathrm{Edd}} \equiv 2 \times 10^{38} \mathrm{erg} \mathrm{s}^{-1}$ and the ignition column depth $y$. We indicate the uncertainty when given, otherwise we assume a $2 \%$ uncertainty. The distances are taken from Christian \& Swank (1997) unless indicated otherwise. Christian \& Swank take the uncertainty in the distance to be 30\%. Jonker \& Nelemans (2004) indicate that the uncertainty in the distances they determined is "probably very large". Therefore we use the $30 \%$ from Christian \& Swank. The uncertainty in the luminosity is dominated by the uncertainty in the distance. Since $L \propto d^{2}$, the uncertainty in $L$ is $60 \%$. As the ignition column depth is proportional to the luminosity (see Eq. (1)), it has the same uncertainty of $60 \%$. In the case of GX $17+2$ this has to be added to the indicated uncertainty in $y$ from the spread in the observed recurrence time.

\begin{tabular}{llllll}
\hline \hline Source & $F\left[10^{-8} \mathrm{erg} \mathrm{s}^{-1} \mathrm{~cm}^{-2}\right]$ & variability & $d[\mathrm{kpc}]$ & $L / L_{\text {Edd }}$ & $y\left[10^{11} \mathrm{~g} \mathrm{~cm}^{-2}\right]$ \\
\hline Sco X-1 & & $13 \%$ & $2.8 \pm 0.3^{a}$ & $0.9 \pm 0.3^{b}$ & $>1.8$ \\
GX 340+0 & $2.67^{c}$ & $15 \%$ & $<11$ & $<1.9$ & $>9.4$ \\
GX 349+2 & $2.45^{d}$ & $16 \%$ & 5.0 & 0.4 & $>1.8$ \\
GX 9+9 & $0.8 \pm 0.012^{e}$ & $14 \%$ & 5.0 & 0.1 & $>0.6$ \\
GX 354-0 & $0.037^{f}$ & $37 \%$ & $4.5^{g}$ & 0.05 & $>0.2$ \\
GX 5-1 & $2.56 \pm 0.4^{h}$ & $8 \%$ & $<9.0$ & $<1.2$ & $>4.4$ \\
GX 9+1 & $2.1^{i}$ & $13 \%$ & $5^{i}$ & 0.3 & $>1.0$ \\
GX 13+1 & $0.803 \pm 0.004^{j}$ & $7 \%$ & 7.0 & 0.2 & $>0.7$ \\
GX 17+2 & $2.26^{k}$ & $12 \%$ & 7.5 & 0.8 & $1.6 \pm 0.8$ \\
Cyg X-2 & $1.3^{l}$ & $23 \%$ & 8.0 & 0.5 & $>2.0$ \\
\hline
\end{tabular}

${ }^{a}$ Distance from parallax measurements (Bradshaw et al. 1999). ${ }^{b}$ From Bradshaw et al. (2003) (2.0-18.2 keV). ${ }^{c}$ From Lavagetto et al. (2004) (0.1-200 keV). ${ }^{d}$ From Iaria et al. (2004) $(0.1-200 \mathrm{keV}) .{ }^{e}$ From observations with BeppoSAX NFI (0.1-200 keV). See Table 3. ${ }^{f}$ From Galloway et al. (2003) (2-60 keV). ${ }^{g}$ Distance from Jonker \& Nelemans (2004) for hydrogen accreting neutron star. ${ }^{h}$ From Paizis et al. (2003) (1.5-40 keV). Note that the flux in the $12-20 \mathrm{keV}$ range is obtained through linear interpolation. ${ }^{i}$ From Iaria et al. (2005) $(0.12-18 \mathrm{keV}) .{ }^{j}$ From Corbet $(2003)$ $(1.5-12 \mathrm{keV})$ and Revnivtsev et al. (2004) (18-60 keV). Flux in 12-18 keV range obtained through linear interpolation. Total energy range: $1.5-60 \mathrm{keV} .{ }^{k}$ From in 't Zand et al. (2004a) (0.1-200 keV). ${ }^{l}$ From Di Salvo et al. (2002) (0.1-200 keV).

\section{Mass accretion rate}

The unstably burning carbon layer to which superbursts are ascribed is formed by the burning of accreted hydrogen and helium. The rate at which the hydrogen and helium are accreted from the companion star plays an important role in whether they burn stably or unstably.

A measurement of the mass accretion rate is most directly done through the X-ray luminosity because the power for the radiation is provided by the liberation of gravitational energy of the accreted matter. However, this is not unambiguous because it is somewhat uncertain what fraction of the liberated energy goes into radiation. It is generally thought that some fraction goes into the kinetic energy of jets. Nevertheless, in the case of neutron star accretors it is not unreasonable to assume that most of the energy goes into radiation (see e.g. Fender 2002).

Another complication involves the determination of the luminosity: distances to LMXBs - needed to translate fluxes to luminosities - are generally poorly known (except for systems in globular clusters). This applies particularly to many of the systems that we study in this paper: persistent LMXBs outside globular clusters without type-I X-ray burst behavior. Systems that exhibit bursts with Eddington-limited fluxes, as diagnosed through photospheric radius expansion, provide a means to find distances with $\approx 30 \%$ accuracy (cf., Kuulkers et al. 2003), and transients that turn quiescent may reveal the optical light of the companion star without contamination by optical light from the accretion disk. Neither of these two conditions apply to most of our systems.

A third complication is that for many systems we do not have a broad-band measurement of the X-ray spectrum that is averaged on timescales longer than that of the typical variability to enable a good bolometric correction. Broadband BeppoSAX $\mathrm{X}$-ray spectra have been taken in many cases but almost never for long enough. This situation may change soon with the results of the deep exposures of INTEGRAL on the Galactic bulge.

We attempted to obtain the best broadband flux measurements for the ten systems here studied, see Table 2. For nine sources this pertains to values found in the literature from RXTE, BeppoSAX and INTEGRAL observations, and for one system, GX 9+9, we perform the analysis ourselves from BeppoSAX data. The data were taken on April 8, 2000, with exposure times of 23, 50 and $22 \mathrm{ks}$ for LECS, MECS and PDS, respectively. Background spectra are taken from blank field observations for LECS and MECS, at the same detector positions, and from offsource pointings with the PDS. We rebin the spectra to limit the oversampling of the spectral resolution to $40 \%$ of the full-width at half maximum, and to obtain at least 15 photons in each spectral bin. During the spectral analysis, a systematic error of $1 \%$ is added in quadrature to the statistical error per spectral bin and the normalization between the three instruments is allowed to vary. We find the generic LMXB spectral model to provide good fits to the data. This model (see e.g. Sidoli et al. 2001) consists of a multi-temperature disk black body (Mitsuda et al. 1984; Makishima et al. 1986) in combination with a comptonized spectrum (Titarchuk 1994; Hua \& Titarchuk 1995; Titarchuk \& Lyubarskij 1995), both absorbed by cold interstellar matter following the model by Morrison \& McCammon (1983). The results of the fit are provided in Table 3.

The flux measurements are based on observations which were typically performed during 1.3 days. From the one-day averaged ASM/RXTE lightcurves we determine the fractional rms variability for each source, see Table 2 . We applied the best distance estimates from the literature and determined the ratio of the resulting luminosity and the Eddington luminosity of a canonical neutron star (i.e. $L_{\mathrm{Edd}} \equiv 2 \times 10^{38} \mathrm{erg} \mathrm{s}^{-1}$ for a neutron star with a mass of $1.4 M_{\odot}$, a $10 \mathrm{~km}$ radius and a hydrogen-rich photosphere). Due to the three sources of uncertainty eluded to above, the resulting ratios are never very accurate, and we are only able to make a crude distinction between the sources close to one tenth and $100 \%$ of the Eddington limit. This inference is in line with the fact that the five sources close to the Eddington limit are the only ones that trace out a Z-shaped curve in a hardnessintensity diagram (e.g., Hasinger \& van der Klis 1989). They are Sco X-1, GX 340+0, GX 5-1, GX 17+2 and Cyg X-2. The others are so-called Atoll sources. 
Table 3. Flux of GX 9+9 from BeppoSAX NFI observations. As model we use an absorbed disk black body in combination with a comptonized spectrum. We provide the parameters from the best fit of the model to the observed spectrum and the unabsorbed $0.1-200 \mathrm{keV}$ flux.

\begin{tabular}{ll}
\hline \hline$N_{\mathrm{H}}$ & $(0.231 \pm 0.003) 10^{22} \mathrm{~cm}^{-2}$ \\
& \\
$k T_{\mathrm{BB}}$ & $0.853 \pm 0.002 \mathrm{keV}$ \\
$\operatorname{Flux}_{\mathrm{BB}}(0.1-200 \mathrm{keV})$ & $(0.33 \pm 0.014) 10^{-8} \mathrm{erg} \mathrm{s}^{-1} \mathrm{~cm}^{-2}$ \\
& \\
$k T_{0}$ & $1.055 \pm 0.003 \mathrm{keV}$ \\
$k T_{\mathrm{e}}$ & $2.458 \pm 0.004 \mathrm{keV}$ \\
$\tau$ & $14.10 \pm 0.06$ \\
Flux $_{\text {comptt }}(0.1-200 \mathrm{keV})$ & $(0.50 \pm 0.04) 10^{-8} \mathrm{erg} \mathrm{s}^{-1} \mathrm{~cm}^{-2}$ \\
$\chi^{2} /$ d.o.f. & $166 / 147$ \\
Flux $(0.1-200 \mathrm{keV})$ & $(0.83 \pm 0.013) 10^{-8} \mathrm{erg} \mathrm{s}^{-1} \mathrm{~cm}^{-2}$ \\
\hline
\end{tabular}

\section{Discussion}

We analyzed a large volume of data from the Wide Field Cameras on BeppoSAX of ten luminous LMXBs to search for superbursts. Except for those already reported from GX 17+2 by in 't Zand et al. (2004a), none were found and we set lower limits on the recurrence time of superbursts that range from 30 to 76 days.

One of the conditions for a superburst to occur is a sufficiently large column depth of accumulated matter. The ignition column depth $y$ for a $1.4 M_{\odot}$ neutron star with a $10 \mathrm{~km}$ radius, assuming a gravitational redshift $z=0.31$, is given by (in ' $t$ Zand et al. 2004a)

$y=2.0 \times 10^{11} \mathrm{~g} \mathrm{~cm}^{-2}\left(\frac{t_{\text {recur }}}{30 \text { days }}\right)\left(\frac{\dot{m}}{\dot{m}_{\text {Edd }}}\right)$,

with $\dot{m}_{\text {(Edd) }}$ the (Eddington limited) mass accretion rate. At a given accretion rate, the lower limit to the recurrence time gives a lower limit to the ignition column depth. Using Eq. (1) we calculate $y$ (see Table 2).

Cumming et al. (2005) investigate several carbon ignition models for superbursts. From these the recurrence time as a function of the mass accretion rate is determined. In Fig. 2 we compare our lower limits to $t_{\text {recur }}$ as well as the observed recurrence times of $4 U$ 1636-536 and GX 17+2 to these models. Looking at what theory predicts, the constraints that we can put on the superburst recurrence time are only meaningful for sources that accrete close to the Eddington limit. For the sources that accrete close to $10 \%$ of the Eddington limit the theory predicts for all models a higher $t_{\text {recur }}$ than our lower limit. Sco X-1, GX 340+0, GX 5-1 and Cyg X-2 accrete matter at a rate comparable to GX $17+2$, namely close to the Eddington limit.

Cumming et al. (2005) discuss the dependence of the ignition of carbon on the heat flux into the superbursting layer from the deeper lying crust: $Q_{\mathrm{b}}$. At a given accretion rate, the recurrence time is lower for higher $Q_{\mathrm{b}}$. Therefore a lower limit to $t_{\text {recur }}$, or alternatively to $y$, gives an upper limit to $Q_{\mathrm{b}}$ for a given model (see Fig. 20 in Cumming et al. 2005). Comparing the lower limit to the ignition column depth of the five brightest sources to the results of Cumming et al. (2005) we find $Q_{\mathrm{b}} \lesssim 0.1 \mathrm{MeV}$ per nucleon for iron composition. However, the large uncertainty in the accretion rate due to the large uncertainty in the distance to these sources prevents us from placing stringent constraints on the models of Cumming et al. (2005).

We found the lower limit to the superburst recurrence time for GX 340+0, GX 5-1 and Cyg X-2 to be about two times the

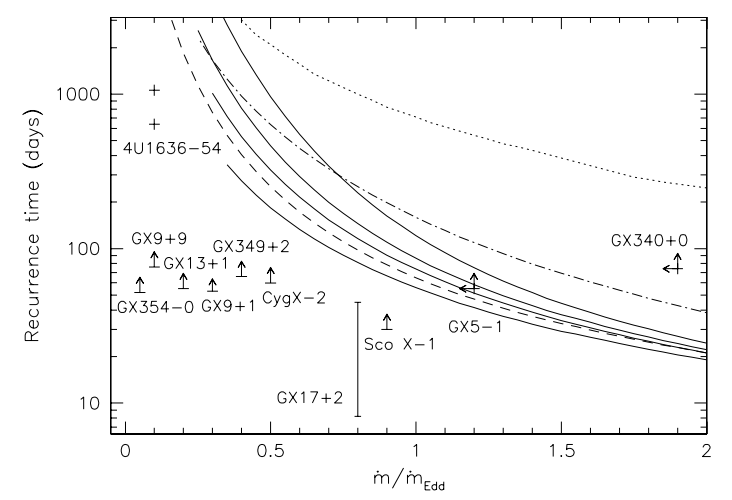

Fig. 2. Superburst recurrence time as a function of the mass accretion rate in units of the Eddington limited mass accretion rate. We show models from Cumming et al. (2005) and observed recurrence times including our lower limits. The solid curves show results for a model with a disordered crust, a composition of ${ }^{56} \mathrm{Fe}$ and a $20 \%$ carbon abundance, without Cooper pairing for four different core neutrino emissivities. The dashed curve is for a model with heavier composition $(A=104)$, the dot-dashed curve has a higher crust conductivity and the dotted curve includes Cooper pair neutrino emission. The curves end at lower $\dot{m}$ where stable carbon burning begins. Note that the uncertainty in the mass accretion rate is at least $60 \%$ for most sources (see Table 2).

recurrence time of GX 17+2. One of our goals was to learn more about the dependence of superbursts on normal bursting behavior. However, we cannot explain the difference in $t_{\text {recur }}$ by the difference in normal type I bursting behavior as both GX 17+2 and Cyg X-2 exhibit bursts, while GX 340+0 and GX 5-1 do not.

From Fig. 2 we see that, depending on the model, a $35 \%$ difference in the mass accretion rate can lead to a two times longer recurrence time. The uncertainty in the mass accretion rate is $60 \%$. Therefore the difference in (lower limit on) $t_{\text {recur }}$ could be due to GX 17+2 accreting faster than GX $340+0$, GX 5-1 and Cyg X-2.

Another possible explanation for the difference in $t_{\text {recur }}$ is that GX 17+2 may differ from the other sources in some aspect which would lower the theoretical curves for this source. The core temperature of GX 17+2 might be higher, which would lead to a lower ignition column depth and therefore a lower recurrence time (see e.g. Fig. 11 in Cumming et al. 2005). Alternatively there may be a difference in the hydrogen and helium abundance of the accreted matter. A larger initial helium abundance will lead directly to a larger carbon yield from rp-process burning (Schatz et al. 2003). Cumming \& Bildsten (2001) find that the amount of carbon required to achieve unstable ignition at accretion rates close to the Eddington limit is approximately $3-5 \%$. The presence of observable superbursts in GX 17+2 might indicate that the accreted material in this source is hydrogen deficient, allowing sufficient carbon to be made, whereas too little carbon is made in other sources to result in a superburst (a similar suggestion is done by Cooper et al. 2006). Another effect, which goes in the opposite direction, is that a lower initial hydrogen abundance will lead to a less extensive rp-process, giving less massive heavy elements, which increases the carbon ignition depth because of the lower opacity of the accumulating fuel layer (Cumming \& Bildsten 2001). However, Brown (2004) shows that this effect is smaller than initially thought. At present only one superburster, the ultracompact system 4U 1820-30, is assumed to accrete hydrogen-poor matter. In this case, the extremely short orbital period of only 11.4 min (Stella et al. 1987) implies that the hydrogen mass fraction is $X \lesssim 10 \%$, consistent with the very luminous and energetic 
superburst observed from this source (Cumming 2003). Another case of a superburster accreting hydrogen-deficient matter is possibly the ultracompact system 4U 0614+091 (see e.g. Nelemans et al. 2004) of which most recently a superburst was observed (Kuulkers 2005).

\section{Conclusion}

We searched for superbursts from ten bright LMXBs. None were found but the four known superbursts from GX 17+2. For the other sources we obtain lower limits to the recurrence time and the ignition column density. Comparing this lower limit for the five most luminous sources, Sco X-1, GX 340+0, GX 5-1, GX 17+2 and Cyg X-2, to the models by Cumming et al. (2005) $Q_{\mathrm{b}} \lesssim 0.1 \mathrm{MeV}$ per nucleon is required. Due to the large uncertainty in the distance to these sources, the uncertainty in the mass accretion rate is large as well. This prevents us from placing firm constraints on the predictions by Cumming et al. (2005). As our search method is indiscriminate of the burst ignition scenario, the recurrence time limits may also be applied to other bursts of similar duration and brightness.

The four luminous non-superbursters have a mass accretion rate comparable to the luminous superburster GX 17+2. For three of these sources we find a lower limit to the recurrence time that is twice as long as the observed average $t_{\text {recur }}$ of GX $17+2$. Most likely this difference is due to GX $17+2$ accreting faster than the other sources and therefore GX 17+2 has a lower $t_{\text {recur }}$. However, we are unable to determine whether this is the case because of the large uncertainty in the mass accretion rate.

We found no superbursts in the six non-bursting sources we considered, which confirms the current observational view of superbursters, i.e. all known superbursters exhibit normal type I $\mathrm{X}$-ray bursts as well. This supports the suggestion that both stable and unstable burning of hydrogen and helium are necessary to produce the fuel for superbursts.

To better constrain models of neutron star interior physics, more long term monitoring observations of (candidate) superbursters are necessary. As superbursts have a long recurrence time, a long exposure time is required for each observed source. The planned mission MIRAX (Braga et al. 2004) will be the most suited for such a task. MIRAX will have on board the spare flight unit of the BeppoSAX WFCs as well as two hard $(10-200 \mathrm{keV}) \mathrm{X}$-ray cameras. The aim of MIRAX is to perform continuous broadband observations of the Galactic center during nine months per year. This will provide a high probability to observe any superbursts that have a recurrence time less than approximately these nine months, which includes the predicted recurrence time of many models shown in Fig. 2 for sources which accrete close to the Eddington limit. The latest observations of superbursts (Remillard et al. 2005; Kuulkers 2005) were done with RXTE/ASM, which observes $80 \%$ of the sky each $90 \mathrm{~min}$. Due to a time resolution of $90 \mathrm{~s}$ the short (1-10 s) burst rise cannot be observed, which makes it more difficult to distinguish between a superburst and a flare. Observations from MIRAX will have a time resolution better than $0.5 \mathrm{~ms}$ which is more than adequate to observe the rise of superbursts.

Acknowledgements. We thank M. Méndez for his useful comments on an earlier version of this paper. We made use of quick-look results provided by the ASM/RXTE team. SRON is financially supported by the Netherlands Organization for Scientific Research (NWO). A.C. acknowledges support from McGill University startup funds, an NSERC Discovery Grant,
Le Fonds Québécois de la Recherche sur la Nature et les Technologies, and the Canadian Institute for Advanced Research.

\section{References}

Belian, R. D., Conner, J. P., \& Evans, W. D. 1976, ApJ, 206, L135 Boella, G., Butler, R. C., Perola, G. C., et al. 1997a, A\&AS, 122, 299

Boella, G., Chiappetti, L., Conti, G., et al. 1997b, A\&AS, 122, 327

Bradshaw, C. F., Fomalont, E. B., \& Geldzahler, B. J. 1999, ApJ, 512, L121

Bradshaw, C. F., Geldzahler, B. J., \& Fomalont, E. B. 2003, ApJ, 592, 486

Braga, J., Rothschild, R., Heise, J., et al. 2004, Advances in Space Research, 34, 2657

Brown, E. F. 2004, ApJ, 614, L57

Christian, D. J., \& Swank, J. H. 1997, ApJS, 109, 177

Cooper, R. L., \& Narayan, R. 2005, ApJ, 629, 422

Cooper, R. L., Mukhopadhyay, B., Steeghs, D., \& Narayan, R. 2006, ApJ, 642, 443

Corbet, R. H. D. 2003, ApJ, 595, 1086

Cornelisse, R., in 't Zand, J. J. M., Verbunt, F., et al. 2003, A\&A, 405, 1033

Cumming, A. 2003, ApJ, 595, 1077

Cumming, A., \& Bildsten, L. 2001, ApJ, 559, L127

Cumming, A., \& Macbeth, J. 2004, ApJ, 603, L37

Cumming, A., Macbeth, J., in 't Zand, J. J. M., \& Page, D. 2005, ArXiv Astrophysics e-prints

Di Salvo, T., Farinelli, R., Burderi, L., et al. 2002, A\&A, 386, 535

Fender, R. 2002, Relativistic Flows in Astrophysics, LNP, 589, 101

Frontera, F., Costa, E., dal Fiume, D., et al. 1997, A\&AS, 122, 357

Galloway, D. K., Psaltis, D., Chakrabarty, D., \& Muno, M. P. 2003, ApJ, 590, 999

Grindlay, J., Gursky, H., Schnopper, H., et al. 1976, ApJ, 205, L127

Hasinger, G., \& van der Klis, M. 1989, A\&A, 225, 79

Hua, X.-M., \& Titarchuk, L. 1995, ApJ, 449, 188

Iaria, R., di Salvo, T., Robba, N. R., et al. 2004, Nucl. Phys. B Proc. Suppl., 132, 608

Iaria, R., di Salvo, T., Robba, N. R., et al. 2005, A\&A, 439, 575

in 't Zand, J. J. M., Kuulkers, E., Verbunt, F., Heise, J., \& Cornelisse, R. 2003, A\&A, 411, L487

in 't Zand, J. J. M., Cornelisse, R., \& Cumming, A. 2004a, A\&A, 426, 257

in 't Zand, J. J. M., Verbunt, F., Heise, J., et al. 2004b, Nucl. Phys. Proc. Suppl., 132,486

Jager, R., Mels, W. A., Brinkman, A. C., et al. 1997, A\&AS, 125, 557

Jonker, P. G., \& Nelemans, G. 2004, MNRAS, 354, 355

Kuulkers, E. 2004, Nucl. Phys. Proc. Suppl., 132, 466

Kuulkers, E. 2005, The Astronomer's Telegram, 483, 1

Kuulkers, E., Homan, J., van der Klis, M., Lewin, W. H. G., \& Méndez, M. 2002, A\&A, 382, 947

Kuulkers, E., den Hartog, P. R., in 't Zand, J. J. M., et al. 2003, A\&A, 399, 663

Kuulkers, E., in 't Zand, J., Homan, J., et al. 2004, in X-ray Timing 2003: Rossi and Beyond, AIP Conf. Proc., 714, 257

Lavagetto, G., Iaria, R., di Salvo, T., et al. 2004, Nucl. Phys. B Proc. Suppl., 132, 616

Lewin, W. H. G., van Paradijs, J., \& Taam, R. E. 1993, Space Sci. Rev., 62, 223 Makishima, K., Maejima, Y., Mitsuda, K., et al. 1986, ApJ, 308, 635

Maraschi, L., \& Cavaliere, A. 1977, in Highlights in Astronomy, ed. E. A. Müller (Dordrecht: Reidel), 127

Mitsuda, K., Inoue, H., Koyama, K., et al. 1984, PASJ, 36, 741

Morrison, R., \& McCammon, D. 1983, ApJ, 270, 119

Nelemans, G., Jonker, P. G., Marsh, T. R., \& van der Klis, M. 2004, MNRAS, 348, L7

Paizis, A., Beckmann, V., Courvoisier, T. J.-L., et al. 2003, A\&A, 411, L363

Parmar, A. N., Martin, D. D. E., Bavdaz, M., et al. 1997, A\&AS, 122, 309

Remillard, R., Morgan, E., \& The ASM Team at MIT, N. 2005, The Astronomer's Telegram, 482, 1

Revnivtsev, M. G., Sunyaev, R. A., Varshalovich, D. A., et al. 2004, Astron. Lett., 30,382

Schatz, H., Bildsten, L., Cumming, A., \& Ouelette, M. 2003, Nucl. Phys. A, 718, 247

Schatz, H., Bildsten, L., Cumming, A., \& Wiescher, M. 1999, ApJ, 524, 1014

Sidoli, L., Parmar, A. N., Oosterbroek, T., et al. 2001, A\&A, 368, 451

Stella, L., Priedhorsky, W., \& White, N. E. 1987, ApJ, 312, L17

Strohmayer, T., \& Bildsten, L. 2003, [arXiv: astro-ph/0301544]

Strohmayer, T. E., \& Brown, E. F. 2002, ApJ, 566, 1045

Titarchuk, L. 1994, ApJ, 434, 570

Titarchuk, L., \& Lyubarskij, Y. 1995, ApJ, 450, 876

Woosley, S. E., \& Taam, R. E. 1976, Nature, 263, 101 